\title{
Enrique Umaña Barragán (1771-1854): Su Conversión de Presunto Sedicioso en Leal Funcionario del Rey (1794-1809) ${ }^{1}$
}

\author{
Enrique Umaña Barragán (1771-1854). His Change from Suspected \\ Seditious Into a Loyal Functionary of The King (1794-1809)"

\section{Enrique Umaña Barragán (1771-1854): Sua Conversão de Presunto Sedicioso em Leal Funcionário do Rei (1794-1809)}

José Antonio Amaya ${ }^{2}$

Universidad Nacional de Colombia - Colombia

jaamay@unal.edu.co

Recepción: 10/02/2013

Evaluación: 12/07/2013

Aceptación: 15/05/2014

Artículo de Reflexión

Una historia crítica es la que nos llama a reflexionar sobre nuestra naturaleza y nuestros límites, sobre las condiciones en las que se estableció lo que entendemos por verdad y por realidad. Una historia crítica perturba y fragmenta, pone de manifiesto la fragilidad de aquello que parece sólido, lo contingente de aquello que parece necesario, las raíces mundanas y cotidianas de aquello que reclama nobleza excepcional. Nos permite pensar en contra del presente, en el sentido de poder explorar sus horizontes y sus condiciones de posibilidad. El objetivo de una historia crítica no es imponer un juicio, sino hacerlo posible.

(Nikolas Rose, 1996).

1 Agradecimientos a la Familia Umaña que nos permitió consultar el AFU. Monsieur Michel Lille contribuyó a la organización y redacción del texto y nos prestó una ayuda invaluable con sus juiciosas, reiteradas y críticas lecturas.

2 Doctor en Historia por l'École des Hautes Études en Sciences Sociales de París. Es Sociólogo por la Universidad Nacional de Colombia. Profesor Asociado de la Universidad Nacional de Colombia, sede Bogotá. Investiga la Historia de las Ciencias Naturales en el Nuevo Reino de Granada de la segunda mitad del s. XVIII y los métodos en Historia. 


\section{RESUMEN}

1794 y 20 de julio de 1810 son dos momentos mayores en la historia política de Colombia. Quienes real o supuestamente se involucraron en el primero no se comprometieron automáticamente con el segundo, y tampoco con la llamada Primera República (1810-1816), como suele narrarlo la historiografía tradicional. Documentos inéditos, ajenos muchas veces a la actividad política propiamente dicha, generados por los llamados próceres en el curso de su contradictoria relación con el Estado absolutista, arrojan luces que señalan nuevos derroteros de interpretación. El caso de Enrique Umaña Barragán es uno de ellos. En estas páginas, Umaña protagoniza su conversión de supuesto conspirador en leal funcionario del rey; años más tarde se consagraráalaadministración desuhacienda Tequendama y en ocasiones extraordinarias ejercerá como funcionario de la naciente República. La defensa, consolidación e incremento de la herencia de sus mayores y el compromiso con sus apellidos definen un destino que supo navegar con prudencia en las aguas revueltas que conducen de la Colonia a la República. La Ilustración francesa lo incita a formarse científicamente, con la autorización y el patrocinio de la monarquía. Hay que precisar, no obstante, la distancia que separa esta adhesión de una inimaginable militancia en las filas de la Revolución francesa en tierras hispanas. Patrimonio y buen nombre dependían de la lealtad a la monarquía. Nuestro prócer definitivamente no encaja en el modelo en que la historiografía tradicional ha querido acomodarlo. Urge una relectura de esta vida para develar el secreto que le permitió franquear incólume y con provecho la ruta de la "antigua a la nueva República".

Palabras clave: Revista Historia de la Educación Latinoamericana, Enrique Umaña Barragán, Republica.

\section{ABSTRACT}

1794 and July 20th of 1810 are two greatest dates in the political history of Colombia.
Those who were involved in the first not committed themselves automatically with the second one in a real or supposed way, nor with the so-called First Republic (18101816), as it is often told in the traditional historiography.Outside the political activity, there were unpublished documents generated by the heros in the course of its contradictory relationship with the absolutist State, this turn on perseptions that indicate new paths of interpretation. One of them is Enrique Umaña Barragán. On these pages Umaña leaded his conversion of a suspected conspirator in a loyal official of the king; years later he was the renowned manager of the farm Tequendama and just sometimes he would work as official of the nascent Republic. The defense, consolidation and increase of the heritage of his biggest and the commitment with his last names defined him a destination that it could sail with prudence in that messed up ocean that they lead from the Colonia to the Republic. The French Enlightenment incited him to be trained in a scientif way, with the authorization and the patronage of the monarchy. Nevertheless, it is necessary to say the distance that separates this adherence of an unimaginable militancy in the lines of the French Revolution in Hispanic grounds. Heritage and good name depended on the loyalty to the monarchy. Our hero definitely did not fit the model that the traditional historiography would like to locate him. It is necessary to re-read his life in order to reveal the secret that he was allowed to pass through unscathed and with advantage the path of the "old to the new Republic".

Key words: Journal History of the Latin American Education, Enrique Umaña Barragan, republic.

\section{RESUMO}

1794 e 20 de Julho de 1810 são os momentos mais marcantes na história política da Colômbia. Os que se envolveram, real ou supostamente, no primeiro não se comprometeram automaticamente com o segundo, e tampouco com a chamada 
Primeira República (1810-1816), como segue narrando a historiografia tradicional. Documentos inéditos, alheios muitas vezes à atividade política propriamente dita, gerados pelos chamados próceres no curso de sua contraditória relação com o Estado absolutista, jogam luzes que assinalam novas possibilidades de interpretação. $\mathrm{O}$ caso de Enrique Umaña Barragán é um desses casos. Nestas páginas, Umaña protagoniza sua conversão de suposto conspirador em leal funcionário do rei; anos mais tarde se consagrará à administração de sua fazenda Tequendama e em ocasiões extraordinárias atuará como funcionário da nascente República. A defesa, consolidação e incremento da herança de seus maiores e o compromisso com seus sobrenomes definem um destino que soube navegar com prudência nas águas revoltas que conduzem da Colônia à República. A ilustração francesa o incitou a formar-se cientificamente, com a autorização e o patrocínio da monarquia. Há que precisar, não obstante, a distância que separa esta adesão de uma inimaginável militância nas filas da Revolução Francesa em terras hispanas. Patrimônio e bom nome dependiam da lealdade à monarquia. Nosso prócer definitivamente não se encaixa no modelo em que a historiografia tradicional quis coloca-lo. Urge uma releitura desta vida para desvelar o segredo que permitiu franquear incólume e com proveito a rota da "antiga à nova República".

Palavras-chave: Revista História da Educação Latino-americana, Enrique Umaña Barragán, república.

\section{INTRODUCCIÓN ${ }^{3}$}

La historiografía identifica en los implicados en los acontecimientos de 1794 a los próceres de la Independencia colombiana y considera que sus actuaciones contribuyeron per se a la creación de la República. Este trabajo critica este punto de vista, con base en la conversión de Enrique Umaña de presunto sedicioso (1794-1799) en corregidor de Zipaquirá (1809-1811), nombrado por el rey Carlos IV y ratificado por la Junta Central y Gubernativa del Reino, en el contexto del expansionismo francés y la derrota de Trafalgar. Además, en estas páginas, se considera la condición familiar de Umaña como hijo, esposo y padre.

Enrique Umaña era un hombre más bien silencioso. El historiador interesado en reconstruir su conversión de presunto sublevado en oficial del rey carece, y por mucho, de fuentes de naturaleza política; además, la correspondencia a disposición es insignificante o poco menos. La mutación política de Umaña es un gran silencio que el historiador debe romper consultando fuentes cuya finalidad no es precisamente la de persuadir al lector. Se trata de sentencias, pasaportes y partidas de diversos tipos, entre otros documentos sin relación natural con el fenómeno de conciencia política que hace el objeto del presente estudio.

Por otra parte, en la reconstrucción de la vida de Umaña se ha venido magnificando su incorporación a la tertulia de Nariño y ulterior participación en los acontecimientos de 1794. Estos episodios se han considerado como el fundamento de la biografía de un republicano probado durante el 20 julio de 1810, durante la Primera República (1810-1816) y la Reconquista (18161819), sin olvidar los servicios que prestó como funcionario a partir de 1819. Este trabajo

3 Un avance de la presente investigación se sociabilizó en el ciclo de conferencias "La Nueva Mirada sobre la Independencia" que organizó en Bogotá la Fundación Santillana para Iberoamérica (Colombia) en asocio con la Universidad Externado de Colombia, del 11 de marzo al 30 de septiembre de 2010. Su redacción se adelantó en el marco del proyecto de investigación "Ensamblado en Colombia: Producción de Saberes y Construcción de Ciudadanías”, financiado por el Departamento Administrativo de Ciencia, Tecnología e Innovación - Colciencias (Código: 220049226305). Diego Armando Varila, estudiante del Departamento de Historia de la Universidad Nacional de Colombia, se desempeñó como asistente durante la fase de redacción. 
considera que el principio organizador de la vida de Umaña no es otro que su condición de primogénito de don Ignacio Umaña Sanabria, propietario de la hacienda de Tequendama, en la sabana de Bogotá. La fugaz vocación de Umaña por el sacerdocio, sus tratos con Antonio Nariño y su entusiasmo por la Revolución francesa y la independencia de los Estados Unidos; su implicación en los acontecimientos de 1794 y su episódico desempeño como mineralogista pueden considerarse como juegos de rebeldía, devaneos literarios, políticos y científicos de alguien que tenía obligaciones ineludibles con el mantenimiento y consolidación del patrimonio de su familia, particularmente al término de la vida productiva de su padre. En las páginas que siguen, Umaña protagoniza sus rebeldías. ${ }^{4}$ Apenas se perfilaba entonces su destino, esto es, la fuerza de la historia y de su familia, que terminará circunscribiéndolo en sus obligaciones de primogénito de hacendado. En este marco, el joven Enrique podía vivir libremente antes de asumir sus responsabilidades heredadas. Su devoción por el universalismo eclesiástico y por el bien de la humanidad, su vehemente deseo de reorganizar el Estado y su anhelo de consagrarle la vida a la historia natural no fueron otra cosa que intentos fallidos de escapar a su destino.

Identificar en Umaña un republicano probado desde la minoría de edad tiene sus riesgos. En primer lugar, impide observar sus retractaciones y probable distanciamiento de los ideales revolucionarios, sus conversiones políticas, ambigüedades y resistencia a poner sus conocimientos científicos al servicio de la revolución de independencia. En pocas palabras, impide ver el tortuoso itinerario de su conciencia política durante la mutación de la Colonia en República. En segundo lugar, puede conducir y ha conducido efectivamente a una deplorable negación de la experiencia espontánea y reiterada, estimando que, a pesar de sus súplicas al rey, de las gracias recibidas, de su enlace matrimonial, arrepentimiento y conversión, Umaña fue siempre y en todo momento un republicano igual a sí mismo. ${ }^{5}$

\section{1794}

El 19 de agosto de 1794 aparecieron fijados en Santafé unos papeles anónimos o pasquines, como fueron llamados entonces. Denunciaban la opresión y usurpación de que se sentían víctimas los neogranadinos, y exigían la eliminación de los estancos. El recuerdo de los Comuneros (1781) y los sucesos recientes en Francia, en particular, la decapitación del rey Luis XVI en 1793, hicieron temer lo peor a las autoridades. Los métodos utilizados para dar con los culpables complicaron aún más las ya de por sí conflictivas relaciones entre criollos y chapetones. Además, se descubrió que Nariño había publicado aquel mismo año de 1794 la Declara-

4 Entre las investigaciones precedentes del autor y en colaboración relacionadas con Enrique Umaña Barragán (EUB), a partir de ahora en las siguientes notas, pueden citarse: José Antonio Amaya, "El Ilustrado santafereño Enrique Umaña Barragán”, en La Familia Umaña, eds. Mauricio Umaña Blanche y Luis Fernando Osorio Umaña (en proceso de publicación); José Antonio Amaya y Diego Armando Varila, "Importancia del procesamiento archivístico de los expedientes del Consejo de Guerra. Caso: Enrique Umaña (1771-1854) (Santafé, Nuevo Reino de Granada, 1816)”, ponencia leída en las XII Jornadas Archivísticas organizadas por la Universidad de Guanajuato y la Red Nacional de Archivos de Instituciones de Educación Superior (RENAIES), Guanajuato, 1315 junio, 2012; Mutis al natural: ciencia y arte en el Nuevo Reino de Granada / Museo Nacional de Colombia, Bogotá, diciembre 2008 - marzo 2009; textos escritos en colaboración con Miguel Ángel Puig-Samper; ed. Ángela Santamaría Delgado (Bogotá: Museo Nacional de Colombia, 2009); "Cuestionamientos internos e impugnaciones desde el flanco militar a la Expedición Botánica", Anuario Colombiano de Historia Social y de la Cultura, no. 31 (2004): 75-118; La traduction espagnole inédite du "Tableau du règne végétal" de Ventenat. Ponencia presentada en el $118^{\circ}$ Congrès national de sociétés historiques et scientifiques (Pau, 2529 octubre, 1993). Les naturalistes français en Amérique du Sud, XVIe-XIXe siècles, ed. por Yves Laissus (París: Ediciones del CTHS, 1995), 167-182; “Enrique Umaña Barragán”, en José María Espinosa. Abanderado del arte y de la patria, catálogo de la exposición abierta en el Museo Nacional de Colombia, Bogotá, 19 octubre, 1994 - 29 enero, 1995; fotografías Julio César Flórez et al. Bogotá, Museo Nacional de Colombia, 1994; Diana Obregón Torres, ed., "Una Flora para el Nuevo Reyno. Las relaciones de Mutis y sus colaboradores con la botánica madrileña (1790-1808)", en Culturas científicas y saberes locales (Santafé de Bogotá: CES, Universidad Nacional de Colombia, 2000), 103-159. Memorias del Tercer Coloquio del Programa Universitario de Investigación (PUI) sobre Ciencia, Tecnología y Cultura.

5 Fue Luis Orjuela, en Minuta histórica zipaquireña (Bogotá: Imprenta de La Luz, 1909), el primero en vislumbrar la conversión de Umaña, aunque se arrepintió en Tributos de Zipaquirá para la Revolución de Independencia (Bogotá: Imprenta de La Luz, 1912). 
ción de los Derechos del Hombre. Se llegó a sospechar que los santafereños querían implantar un sistema similar al francés en el virreinato. ${ }^{6}$

Se iniciaron entonces tres procesos: por los "Derechos del Hombre", los Pasquines y por Sedición. Los culpables de los dos primeros fueron condenados un año después. A Nariño se le aplicaron 10 años de presidio en África, aunque escapó a su arribo a Cádiz. Los Pasquineros, por su parte, fueron enviados a España y luego a África. Los implicados en el delito de sedición fueron Francisco Antonio Zea, José María Cabal, Sinforoso Mutis, Luis de Rieux, Manuel Froes, José Ayala, Ignacio Sandino, Pedro Pradilla, Bernardo Cifuentes y Enrique Umaña. Las razones invocadas para arrestarlos nunca fueron claras, tanto así que, a pesar de ser enviados a España bajo partida de registro y permanecer allí hasta 1799, el proceso formal no se inició y terminaron recibiendo compensaciones de la corona.

Aunque aquí no cabe un balance historiográfico de los sucesos de 1794, sí puede decirse que algunos investigadores coinciden al señalar su importancia, aunque con matices distintos. Para algunos, 1794 inaugura el proceso de independencia en América, que terminará 30 años después con Sucre en Ayacucho. Forero Benavides, Ibáñez y König lo asocian con la Revuelta de Los Comuneros. Para ellos, los dos acontecimientos constituyen una clara muestra de la inconformidad de los criollos con la manera en que los españoles gobernaban el Nuevo Reino. Posada destaca el revuelo que los sucesos generaron en la capital; sin embargo, considera que las conexiones de 1794 con la Independencia no son fáciles de establecer, a pesar de querer conocer el papel que desempeñaron algunos de los involucrados en la conducción ulterior de la república. Por su parte, Hernández de Alba ve en 1794 la consecuencia de la Ilustración, que había llenado de expectativas a jóvenes estudiantes, ansiosos con las noticias que llegaban de Francia y Estados Unidos. Para Silva, la prisión que sufrieron los presuntos sediciosos se convirtió en ocasión de un viaje de estudio. ${ }^{7}$

Todos coinciden en que 1794 marcó un punto de no retorno en las relaciones entre criollos y peninsulares. La desmesurada reacción de las autoridades españolas, representantes del rey, ante una serie de hechos aislados, que investigaciones probaron no tener relación mutua, incrementó el resentimiento de la élite criolla que no volvió a ver con los mismos ojos a los chapetones.

Aunque no fueron formalmente acusados, Umaña y sus compañeros vivieron cerca de 5 años de cárcel en Santafé (septiembre de 1794 - noviembre de 1795) y Cádiz (18 de marzo

6 "Lo menos que se decía era que todos los criollos eran unos herejes y sublevados; que habían adoptado las máximas de la Francia y trataban de sacudir el yugo del soberano. Por desgracia, el Colegio del Rosario -la casa más virtuosa de Santa Fe- ha sido el más maltratado y calumniado, hasta el extremo de decir que se hacían en él Juntas de Sublevación presididas por su rector D. Fernando Caicedo (que es un juiciosísimo eclesiástico), adonde concurrían los sujetos más honrados y visibles del lugar ... Yo -que a la circunstancia de vivir en el Colegio, añadía la de entender el francés, que ya muchas gentes de aquí lo reputan como delito y basta en el día para hacer a un hombre sospechoso, y la de ser amigo de [Francisco Antonio] Zea -temí por instantes mi prisión, pero por fortuna quiso Dios que todo terminase en el escrutinio de mis libros y papeles en que no se halló (por confesión del mismo Juez que lo practicó, en la diligencia extendida en su virtud) la menor cosa, ni vestigio de cosa sospechosa". En "De una carta de D. Camilo Torres a su padre D. Jerónimo Francisco de Torres, en la cual le informa de los sucesos políticos de Santa Fe en 1794", El Correo Nacional, 21 de julio, 1894. Atención del abogado Luis Fernando Osorio Umaña.

7 Anónimo, “1794-1894”, Los Hechos Serie 2 No. 156, 20 de julio,1894: 678; Abelardo Forero Benavides, "La incubación de la Independencia”, en Impresión y represión de los Derechos del Hombre, ed. Abelardo Forero Benavidez (Bogotá: Ediciones Universidad de los Andes, 1967), 121-138; Pedro María Ibáñez, "La conspiración de 1794", Lecturas Populares, El Tiempo, Serie 3, no. 30, no. 984 (1914); Hans-Joachim König, En el camino hacia la nación. Nacionalismo en el proceso de formación del Estado y de la nación de la Nueva Granada, 1750 a 1856 (Bogotá: Banco de la República, 1994); Eduardo Posada, "El proceso de los pasquines", Boletín de Historia y Antigüedades 8, no 96 (1913): 721-728; Guillermo Hernández de Alba, "Herencia y gloria acrecentadas. Estudiantes y profesores de 1794", El Tiempo, 7 de julio, 1957; Renán Silva, Los ilustrados de Nueva Granada 1760-1808. Genealogía de una comunidad de interpretación (Medellín: Banco de la República, Fondo Editorial Universidad Eafit), 2002. 
de 1796 - 28 de agosto de 1799). ${ }^{8}$ El Consejo de Indias ordenó su libertad, de modo que quedaron hábiles para "continuar sus estudios y profesión sin nota y como si no se hubiese procedido en contra de ellos". Umaña tuvo la "satisfacción" de "penetrar de su inocencia el corazón" del monarca, durante una audiencia que se le acordó a principios de $1800 .{ }^{9}$ Al declarar inocente a Umaña, la corona reconocía haber cometido una injusticia que era indispensable resarcir, sin embargo, que nada podía compensar los daños ocasionados al sindicado y su familia. Baste decir que Umaña fue encarcelado a los 23 años siendo menor de edad; en lenguaje de la época, el niño Enrique había pasado a ser el joven Umaña en prisión. Un aura de suspicacia lo estigmatizará el resto de sus días. ${ }^{10}$

Enrique era el primogénito del matrimonio constituido por Ignacio Umaña Sanabria con Isabel Barragán Gaitán. Al momento de ser privado de la libertad, el damnificado había concluido sus estudios de Derecho y comenzaba sus prácticas. La minoría de edad del hijo hacía responsable al padre de su defensa y gastos durante el presidio. ${ }^{11}$ En Santafé, Don Ignacio contrató los servicios del procurador Luis de Ovalle, quien abandonó la causa intimidado por las autoridades; en seguida, ningún abogado local quiso ocuparse del caso. Obligado a asumir personalmente la defensa, el padre suplicó sin éxito directamente al rey. Cuando Enrique fue trasladado a Cádiz, Saturio Ángel Velasco, solicitador en Madrid, asumió su defensa. No obstante las suspicacias, los negocios de los Umaña continuaron tan productivos como antes de 1794, a pesar de las deudas contraídas en aquellas circunstancias; incluso se observa durante estos años un incremento de las tierras de la familia. ${ }^{12}$

8 Diana Soto Arango, Francisco Antonio Zea. Un criollo ilustrado (Madrid: Ediciones Doce Calles, Colciencias, RUDECOLOMBIA, 2000), 131, nota 3 .

9 José Manuel Pérez Sarmiento, ed., Causas célebres a los precursores. "Derechos del Hombre”, Pesquisa de Sublevación, Pasquines Sediciosos, 2 vols. (Bogotá: Imprenta Nacional, 1939): 2: 230. Por otra parte, en Orjuela, Tributos de Zipaquirá, 92 y 192, se afirma erróneamente que Enrique Umaña se "fugó del hospital de Cádiz".

10 En carta a destinatario desconocido escrita en octubre de 1800, Umaña alude a la inconformidad que produjo en algunos sectores de Santafé la sentencia citada del Consejo de Indias. "Basta con lo que he conseguido y lo que han conseguido mis compañeros [se refiere a los conocimientos científicos adquiridos en Madrid por él y por Zea y Cabal] para contentar las preocupación [e]s de algunos tontos de nuestros paisanos que han juzgado poco satisfactoria la resolución de nuestra causa". Original en Archivo de la Familia Umaña (AFU). Hacia 1800, la palabra preocupación era sinónima de prejuicio. Entre los inconformes con el indulto se encontraban ciertamente los militares o, al menos, un sector de ellos. El "Plan de un cuerpo militar de ingenieros mineralógicos" [Santafé, 1802] consideraba, por ejemplo, que el desconocimiento del derecho del cuerpo castrense para asumir la dirección de las casas de moneda de Santafé y Popayán, las fábricas de salitre y pólvora y las salinas de Zipaquirá era resultado de la “infección de las máximas del pseudo-político Maquiavelo ..., de ingratitud y ... de todos los vicios y delitos atroces que avergüenzan a la humanidad", refiriéndose sin duda a los implicados en los sucesos de 1794. Véase José Antonio Amaya, "Cuestionamientos internos e impugnaciones desde el flanco militar a la Expedición Botánica”, Anuario Colombiano de Historia Social y de la Cultura, no. 31 (2004): 95. El primer interesado en correr una cortina de humo sobre los incidentes de 1794 era el propio Umaña, quien en su Relación de méritos evita cuidadosamente cualquier alusión a aquel incidente, dando a entender que su primer viaje a España había sido voluntario ("habiéndose venido a estos Reynos", peninsulares). Enrique Umaña Barragán, Relación de los méritos y ejercicios literarios del doctor don EUB. Madrid, 14 de mayo, 1807

11 EUB y los "procesados demostraban que formaban parte de la elite criolla y eran personas 'acostumbradas a la decencia, y a la abundancia' que ahora se veían sometidos a 'morir de hambre o de las enfermedades, porque los seis reales diarios que les daban no alcanzaban' ni para comer un mal cocido cada veinte y cuatro horas. Sin embargo, cuando fueron declarados en libertad siguieron solicitando los citados seis reales hasta tanto no se les abonara el 'costo de la embarcación hasta sus casas', porque se encontraban en estado 'lamentable”'. Oficio al Consejo de Estado, Cádiz, 30 de abril de 1798, y Oficio de los reos residentes en Cádiz, 2 de septiembre de 1799, Archivo Histórico Nacional (España), Consejo, leg. 21.249, f. 4 y leg. 21.236, citados por Diana Soto Arango, Francisco Antonio Zea, 86-87. Al respecto, EUB declarará ante el Libertador Presidente de la República desde Santafé en 1828: "Fácil es percibir cuánto padecerían mis padres viéndome víctima de tan furiosa persecución, cuántos gastos harían para aliviar algo mis trabajos, y que esto en tanto tiempo debió disminuir mucho su fortuna y p[o]r consiguiente mi patrimonio y el de mis nueve hermanos". Archivo General de la Nación (AGI), República, Peticiones y solicitudes, leg. 9, ff. 824-826.

12 Una revisión (1760-1810) de la Notaría Segunda de Santafé en el AGN indica que, entre 1794 y 1798, Ignacio Umaña Sanabria y su hijo Ignacio Umaña Barragán contrajeron no menos de 4 préstamos con comerciantes y hacendados. El padre obtuvo uno de ellos -8 de abril de 1795- de Pedro Rodríguez, por 1440 pesos en moneda de plata usual y corriente a pagar a 4 años, con un rédito anual del $5 \%$, dando en garantía la hacienda de Tequendama. El préstamo se canceló el 17 de mayo de 1808 (AGN, Notaría Segunda de Santafé, tomo 188, ff. 122-123; comunicación de don J. Vladimir Torres Moreno, 2 de febrero, 2011). Por otra parte, durante la ausencia de EUB, su padre adquirió la hacienda de Junca y las tierras Trapiche y demás conocidas con el nombre de Úrsula Núñez, estas últimas ubicadas en la Parroquia del Colegio, jurisdicción de la Mesa de Juan Díaz (Cundinamarca). La compra de Trapiche y Úrsula Núñez ocurrió en 1800; la de Junca, antes de 1804. El valor de las primeras ascendió a 1550 pesos; el de la segunda, a 1500 pesos. 
Con este trasfondo, Enrique Umaña suplicó la dispensa de 2 años de prácticas que le faltaban para recibirse de abogado. La Real Orden de 4 de mayo de 1800 aprobó la solicitud. Umaña se convirtió -según lo consignó en el borrador de su tesis- en el primer criollo en sustentar una tesis doctoral ante el Consejo de Indias. El 9 de septiembre de 1800, se le "instaló de abogado", quedando habilitado para litigar en calidad de jurisconsulto del propio Consejo y de todas las audiencias, tribunales y juzgados de los reinos y provincias de Nueva España, Perú y Nueva Granada. En el Nuevo Reino, ningún abogado contaba con un ámbito de ejercicio tan extendido, ni siquiera José Félix de Restrepo (1760-1832) o Camilo Torres Tenorio (1766-1816), eminentes letrados con privilegio de litigar en las audiencias de Santafé y Quito. Así, a los 29 años, la condición de abogado le abrió efectivamente a Umaña las puertas de la edad adulta. Sin embargo, el título era más bien honorífico, pues a poco de recibirlo, el rehabilitado comunicó al rey que su verdadera vocación era la historia natural. Puede decirse que a Umaña le repugnaba la jurisprudencia, aunque aquí se impone la prudencia, pues no cabe duda de que él sabrá explotar sus conocimientos en el debate para limitar el fuero eclesiástico y someter a los sacerdotes a la justicia ordinaria en casos de delitos comunes, por ejemplo, sin olvidar los negocios de su familia y de los suyos en general.

En 1800, los neogranadinos fueron acogidos en Madrid por el abate y botánico Antonio José Cavanilles (1745-1804) y no por Casimiro Gómez Ortega (1741-1818), este último primer profesor, desde 1771, del Real Jardín Botánico. Aunque el Jardín nunca fue propiamente hablando la institución de tutela de la Real Expedición Botánica del Nuevo Reino de Granada (1783-1816), ${ }^{13}$ las comunicaciones entre los dos centros se hallaban rotas o poco menos desde 1784. Corresponsal de Mutis desde 1786 y de Zea desde 1798, Cavanilles le había declarado una formidable oposición a Gómez Ortega desde 1787, cuando fracasó en su intento de acceder a la dirección del Jardín. ${ }^{14}$ En lo más agudo de la controversia, Zea y sus adeptos, Umaña y Cabal, se alinderaron del lado de Cavanilles. ¿Dónde estaban las preferencias del gobierno? Baste señalar que 6 títulos de botánica publicados por Cavanilles entre 1790 y 1796 salieron de la Imprenta Real. Entre 1790 y 1800, Gómez Ortega, por su parte, tuvo que publicar en imprentas particulares diez títulos de botánica, agricultura y medicina. ${ }^{15} \mathrm{~A}$ su vez, con la subida al poder (1787-1790) de Antonio Porlier (1722-1813), Marqués de Bajamar, se acabaron los años dorados del americanismo (1776-1787), auspiciados por el Ministro de Indias José de Gálvez y Gallardo (1720-1787), marqués de Sonora. El Ministerio de Indias desapareció para integrarse a la Secretaría de Gracia y Justicia de Indias. La llegada, primero, del conde de Aranda y, después, de Godoy, a la Primera Secretaría de Estado trajeron consigo un cambio notable en el apoyo institucional hacia la botánica española. Los aspectos fármaco-terapéuticos perseguidos por el grupo de Gómez Ortega y apoyados por el conde de Floridablanca, ya no serían defendidos por Godoy, mucho más proclive a la escuela botánica encabezada por Cavanilles. ${ }^{16}$ Por otra parte, en un momento de exacerbados antagonismos entre la tradición

13 Véase José Antonio Amaya y J. Vladimir Torres Moreno, "La Expedición Botánica a la luz de las cifras”, en Ensamblando Estados, ed. Olga Restrepo Forero (Bogotá: Colciencias, 2013): “Gómez Ortega se rehusó a redactar las instrucciones para la Expedición Botánica del Nuevo Reino de Granada, dejando vacía su dirección científica. En la ocurrencia, el arzobispo-Virrey Antonio Caballero y Góngora debía atenerse a las de la Expedición al Perú, que no eran por cierto del agrado de Mutis. Sin embargo, nunca se ha encontrado este documento, si alguna vez existió. En estas condiciones, la Expedición nació carente de directivas explícitas para su funcionamiento científico y administrativo. Habiendo dejado Gómez Ortega a Mutis en 'libertad' para organizar la Expedición, ésta encontró en su Director su propia autoridad de regulación".

14 Antonio González Bueno, Antonio José Cavanilles (1745-1804). La pasión por la ciencia (Madrid: Fundación Jorge Juan, 2000$), 15$.

15 José María López Piñero y María Luz López Terrada, Bibliografía de Antonio José Cavanilles (1745-1804) y de los estudios de su vida y su obra, 3a ed. rev, s. l., s. e., s. f., http://www.uv.es/rseapv/Publicaciones/Cavanilles/ RSEAP_Cavanilles_2A.pdf; Biblioteca Nacional de España, Catálogo de la biblioteca: http://catalogo.bne.es/uhtbin/webcat; sitio Web del Real Jardín Botánico de Madrid: http:// www.rjb.csic.es/jardinbotanico/jardin/

16 Raúl Rodríguez Nozal, "La Oficina Botánica (1785-1835). Una institución dedicada al estudio de la flora americana”, Asclepio 47, no. 2 (1995): 172. http://asclepio.revistas.csic.es/index.php/asclepio/article/ viewArticle/442. 
y las novedades, Cavanilles apoyaba las medidas favorables a la Ilustración, mientras la represión buscaba evitar a la monarquía el contacto con las ideas revolucionarias. ${ }^{17}$ Godoy -afrancesado como Cavanilles- regresó al cargo de Ministro Principal justo en 1800. Desde la subida al poder del llamado Príncipe de la Paz en 1792, Cavanilles acarició la posibilidad de realizar todos sus ambiciosos sueños. ${ }^{18}$

Así las cosas, aquel mismo año de 1800 Umaña solicitó permiso para ingresar al Real Gabinete de Mineralogía. La petición sugiere un nuevo gesto de rebeldía. Tras 5 años de ausencia, el recién liberado no parecía tener prisa para regresar a Santafé, abrazar a sus padres y vincularse a la administración de Tequendama. El permiso concedido confirma la confianza que ahora inspiraba Umaña en las autoridades, pues la Química y la Mineralogía asociadas brindaban conocimientos estratégicos para la seguridad del Estado: amonedar, fabricar armas y producir pólvora. Por primera vez, a sus 29 años, el héroe de esta historia se comportaba como un individuo que hacía lo que le gustaba -o más bien, suplicaba lo que decía gustarle-, apoyado por sus padres, que continuaban sufragando sus gastos, a pesar de que los mineralógicos estudios poco y nada tenían que ver con la administración de Tequendama, productora de carne y trigo. Ahora Umaña bien podía sentirse no sólo resarcido sino favorecido por la monarquía.

Tras una breve permanencia en Madrid, los rehabilitados marcharon a perfeccionarse en el Instituto Nacional de Francia. Una vez más, Umaña contó con el apoyo de sus padres, interesados en redorar el apellido con los viajes y diplomas del hijo. Por aquel entonces, las relaciones políticas tenían dificultades extremas, las mismas con las cuales la corte de Madrid se había debatido durante el siglo XVIII. España se encontraba entre Inglaterra, deseosa de ampliar su influencia sobre el Imperio de América, y Francia, que representaba un aliado natural aunque interesado. El gobierno de París deseaba reparar los excesos del comercio nacional sirviéndose de la flota española; unida a la francesa, esta parecía equilibrar la de Inglaterra. ${ }^{19}$ El reinado (1788-1808) de Carlos IV (1748-1819), por su parte, se propuso continuar la renovación interior y mantener la alianza con Francia, evitando escrupulosamente cualquier sujeción, mejorar la administración de América y ponerla al abrigo de las ambiciones comerciales británicas, tratando de borrar el ejemplo de la Independencia de los Estados Unidos. ${ }^{20}$ Además, desde 1795, el tratado de Basilea había puesto las relaciones entre España y Francia en una situación análoga a la de antes de la ruptura en 1793. El Segundo Tratado de San Ildefonso (1800) reafirmó esta orientación de entendimiento con París.

Estando en París, Zea vinculó a Umaña y a Cabal a un ambicioso "Proyecto de reorganización de la Expedición Botánica", que quedó listo entre 1801 y 1803. ${ }^{21}$ Los acusados de segregacionismo se proponían ahora introducir la Agricultura, la Química y la Mineralogía en la Expedición Botánica, con el apoyo de Godoy y la improbable anuencia de Mutis. Ahora que Cavanilles se desempeñaba como Primer Profesor del Jardín, desde el 17 de junio de 1801, la Expedición de Santafé, representada en este caso por Zea y sus adeptos, comenzaba a conocer una nueva época en sus relaciones con Madrid. Se buscaba asociar las nuevas modalidades del resarcimiento con el intento de renovar el centro santafereño. Los criollos se mostraban dispuestos a implementar en Santafé la política centralista trazada por Cavanilles. El Real

\footnotetext{
17 Yves Bottineau, Les Bourbons d'Espagne 1700-1808 (París: Fayard, 1993), 339.

18 José María López Piñero, La obra botánica de Cavanilles (Valencia: Faximil Ediciones Digitales, 2004), 73. http://www.faximil.com/ descargas/estudioicones.pdf

19 Bottineau, Les Bourbons, 345.

20 Bottineau, Les Bourbons, 331.

21 Un análisis detallado de este proyecto aparece en Amaya, “Cuestionamientos internos", 75-118.
} 
Jardín debía convertirse en el centro de los demás jardines de la Península y de los que existían o pudiesen crearse en dominios españoles. Estos debían enviar cada año al de Madrid un estado de las plantas vivas que tenían, del herbario, biblioteca, enseñanza y discípulos, otro de los fondos y gastos, y una relación de los desembolsos efectuados y de las obras que quisieran imprimir. Ahora, como en 1783, cuando se creó la Expedición Botánica, la monarquía se servía de este centro para introducir las Ciencias naturales en el Nuevo Reino, en círculo restringido y sin enseñanza pública, alternativa más expedita y económica que la de reformar la universidad, según planes postergados desde los años 1760. La Expedición era un "objeto científico" que contribuía a excusar la aprobación gubernamental de la enseñanza científica en la formación de los criollos. Quienes desearan completar su formación debían viajar a la metrópoli. Por otra parte, la conducción a Madrid del codiciado gabinete de Mutis figuraba ciertamente entre los compromisos adquiridos por Zea. ${ }^{22}$

Así se desplazaban de la política a la ciencia los ideales reformadores de Umaña, quien acariciaba la idea de integrarse al real servicio, con la misión de fundar la sección de mineralogía de la Expedición. La perspectiva de convertirse en funcionario convenía al interesado tanto como a sus gentes, que continuaban banarrastrando el estigma de la traición al rey. Sin embargo, el plan de Zea se tornó imposible o casi en 1803, cuando su autor fue nombrado segundo profesor del Real Jardín. ${ }^{23} \mathrm{Ni}$ Cabal ni Umaña retomaron el proyecto.

La Corona había dispuesto en 1801 acordarle a Umaña quince mil reales vellón ( $\approx 973$ pesos) para que retornara a su patria provisto con una colección de mineralogía. Umaña regresó a Santafé en 1802 y se vinculó a la Expedición hacia abril de 1803, sujetándose sin chistar a la firme política autonomista de Mutis. Una vez más, los Umaña subvencionaron los viajes de Enrique por el virreinato, ${ }^{24}$ así como las correspondencias que, por aquel entonces, el hijo cultivó con los científicos franceses Étienne Pierre Ventenat (1757-1808) y René Just Haüy (1743-1822). La vinculación de Umaña a la Expedición hizo pública su rehabilitación.

El 4 de junio de 1804, el Cabildo Secular de Santafé postuló a Umaña para que gestionara en Madrid la creación de una escuela de mineralogía adscrita a la Expedición Botánica. El "Informe" correspondiente daba cuenta de la "irreprensible conducta" del postulado. ${ }^{25}$ Se trataba de la más alta responsabilidad científica del Nuevo Reino, "cuya principal riqueza consistía en muchas y abundantes minas, y no haber allí ninguno de los conocimientos necesarios para beneficiarlas con utilidad".

Con esta misión, Umaña emprendió su segundo y último viaje a Europa (1804-1809), financiado una vez más por su familia. Además, tenía la intención de casarse, conseguirle colegio a su hermano León José Umaña (n. 1789), conducir a Madrid 300 quintales de quina e importar con el producto de la venta mercaderías por un valor equivalente. El negocio de las quinas por Umaña estaba sin duda en relación con los herederos del poderoso comerciante toledano

$22 \mathrm{Al}$ respecto existe el antecedente de que cuando la expedición comandada por Alejandro Malaspina retornó a España, Cavanilles solicitó al duque de la Alcudia, en marzo de 1795, el libre acceso a los materiales botánicos recogidos. Godoy no aceptó porque Malaspina argumentó "el agravio que se hacía a éstos en que otro publicase antes ... las plantas de vidas a su zelo y a los trabajos de su peregrinación .... González, José Antonio Cavanilles, 195.

23 Tras la muerte de Cavanilles, en 1804, Godoy nombró a Zea director del Real Jardín, marginando a todos los demás naturalistas, a pesar de que Zea sólo había publicado un artículo sobre la quina para halago de Mutis. López, La obra botánica, 74.

24 "Luego que llegó a Santafé de Bogotá se agregó voluntariamente a la Real Expedición Botánica de aquel Reino, trabajando en ella sin gajes ni retribución alguna, y habiéndose propuesto trabajar en la descripción del reino mineral de aquellas provincias, emprendió a sus expensas algunos viajes dirigidos a tomar conocimiento de la mineralogía de aquellos territorios, visitando alunas de sus minas". Umaña, Relación de méritos.

25 Ibíd. 
Gregorio Sánchez Manzaneque (1732-1800), distribuidor en Madrid de las quinas de la provincia de Santafé, ${ }^{26}$ avaladas por Mutis. La competencia de los Sánchez Manzaneque no era otro que el propio Gómez Ortega, que distribuía en su madrileña botica las quinas del Perú.

La prometida de Umaña, María Vicenta Sánchez Manzaneque (1783-1849), era hija de Gregorio y de la dama santafereña María Luisa Pérez Delgado. ${ }^{27}$ Tras hacer carrera pública y fortuna en el Nuevo Reino, Sánchez Manzaneque se trasladó a Madrid en 1785. Por otra parte, uno de los hermanos de la novia, Juan Manuel, ostentaba el grado de capitán de la Real Compañía de Guardias Valonas, un ejército de nobles creado para proteger al rey. Los Sánchez Manzaneque estaban ciertamente convencidos de la inocencia de Enrique Umaña; en todo caso, hicieron gala de liberalidad integrándolo al seno de su familia. El matrimonio introdujo entre los Umaña un componente peninsular que resultaba novedoso en esta estirpe de criollos que, a lo largo del siglo XVIII, no conoció alianzas con peninsulares. El héroe de esta historia conoció la paternidad en 1805, con motivo del nacimiento en Madrid de Enrique Benito Umaña Manzaneque (†1876).

Las consecuencias de la derrota de Trafalgar ocurrida el 21 de octubre de 1805 fueron inmensas. La elite de los oficiales de la Armada pereció. La Marina Real no vio su fin, pero recibió un golpe severo. Las relaciones entre España y América se rompieron. El dinero de América no llegaba a la metrópoli para alimentar las cajas reales. Los barcos mercantes quedaban a la merced de los corsarios británicos. Napoleón, por su parte, continuaba ganando poder en Europa. En estas circunstancias, el rey Carlos IV quedó prisionero entre las exigencias de Napoleón y las brutalidades de Inglaterra. ${ }^{28}$

Semejantes condiciones imposibilitaron la creación de la escuela de mineralogía y persuadieron a Umaña a "solicitar", en 1807, la dirección de las salinas de Zipaquirá, haciendo "presente a Su Majestad el atraso en que estaban por falta de buena dirección" ${ }^{29}$ La administración de las salinas dependía del Corregidor de Zipaquirá. ${ }^{30}$ El monarca accedió a la tímida petición, aunque por Real Cédula de 29 de agosto de 1807 determinó conferirle a Umaña el propio el corregimiento de Zipaquirá:

Don Carlos IV ... por cuanto habiendo cumplido su tiempo don Agustín Vargas de Novoa, actual corregidor de Zipaquirá en el virreinato de Santafé, ha venido en consulta de mi Consejo de Cámara de Indias del corriente año en conferir dicho empleo a vos don Enrique de Umaña, abogado de dicho mi Supremo Consejo y Reales Audiencias de Indias. Por tanto, quiero y es mi voluntad que entréis inmediatamente

26 Comunicación del Doctor Félix Muñoz Garmendia, Real Jardín Botánico (Madrid), abril, 2009.

27 Don Gregorio era pariente consanguíneo de don Andrés Mayoral, arzobispo de la Santa Iglesia de Valencia. Además, María Vicenta era sobrina de don Francisco Sánchez Mayoral, administrador general y juez conservador privativo de la Encomienda de la Villa de Segura de la Sierra, posesión del infante don Luis Antonio Jaime de Borbón (1727-1785), hijo del rey Felipe V (1683-1746). Entre los Sánchez Manzaneque, se contaban "muchos eclesiásticos y comisarios del Santo oficio", así como varias personas que habían obtenido los oficios honoríficos de la República. Hacia 1772, la familia contaba seis sacerdotes en Orgaz. La notoriedad de estas gentes procedía sobre todo de los apellidos De La Serna y Manzaneque, familias muy antiguas en la villa de Orgaz, reinado de la ciudad de Toledo. AFU, Informaciones de genere, moribus y vita, presentada por Gregorio Sánchez Manzaneque, teniente de Milicias de Santafé ante el alcalde ordinario de la misma ciudad, a efectos de que se anexen a las existentes en el Archivo del Cabildo, Santafé, 27 de octubre, 1772.

28 Bottineau, Les Bourbons, 162.

29 Amaya y Varila, "Importancia del procesamiento".

30 Antonio García de la Guardia, Kalendario manual y guía de forasteros en Santafé de Bogotá, capital del Nuevo Reyno de Granada, para el año de 1805 (Santafé: Imprenta Real, 1804), 112-114, 212; Antonio García de la Guardia, Kalendario manual y guía de forasteros en Santafé de Bogotá año de 1806 (Bogotá: Edición facsimilar, Banco de la República, 1988), 227. 
a ejercer el expresado corregimiento de Zipaquirá y que le sirváis por espacio de cinco años. ${ }^{31}$

Por aquel entonces, los Gobernadores, los corregidores y los tenientes letrados representaban los altos cargos políticos en el Nuevo Reino. La prestancia del corregidor de Zipaquirá dimanaba del hecho de ser nombrado directamente por el rey. Además, después del de Santafé, el corregimiento de Zipaquirá era el más importante dentro de la provincia de Santafé, ${ }^{32}$ aunque tenía una asignación más bien simbólica. "El Corregimiento de Zipaquirá es un destino infeliz que no tiene emolumento alguno, ni otro sueldo que el de 400 pesos anuales, por el agregado de Juez conservador de las salinas que allí se administran por cuenta del Rey". ${ }^{33}$ El corregidor de Zipaquirá desempeñaba funciones políticas, judiciales, de escribanía y en relación con la administración de las salinas.

El nombramiento demostraba que el gobierno tenía capacidad de integrar en su aparato burocrático a un presunto sublevado declarado inocente tras cinco años de prisión, con un perfil de abogado-mineralogista formado en Europa, excepcional incluso en España. La comunidad de abogados de la Nueva Granada colonial, así como las de otras regiones del Imperio español, incluía a muchos de los candidatos más calificados y aspirantes más ansiosos a los cargos públicos, habiéndose convertido el abogado-burócrata, a secas, en una figura central del sistema colonial. Sería necesario poner el nombramiento de Umaña en perspectiva, por cuanto los Borbones buscaron desalojar a los criollos de los altos cargos de la burocracia indiana. En ese sentido, Umaña también fue una excepción, pues desde 1792 el corregimiento de Zipaquirá había venido sido confiado exclusivamente a peninsulares: Jerónimo de Mendoza y Hurtado (1792-1795), Manuel de Villaroel y Vizconde (1795-1802) y Agustín Vásquez de Novoa (1802-1809).

Una vez nombrado Umaña en 1807, el Ministro de Hacienda Cayetano Soler (1746-1809) lo envío a Francia para que conociera los últimos adelantos en el ramo de las salinas. Camino de París, matriculó a su hermano León José, de 18 años, en el colegio de Sorèze, en el sur de Francia, a unos $80 \mathrm{~km}$ de Toulouse. A principios del siglo XIX, Sorèze formó militares, filósofos y políticos de la aristocracia europea y estadounidense. La elección sugiere la antipatía que inspiraba en el acudiente Napoleón y la Revolución francesa. En este sentido, el criollo L. J. Pontón se refería con tonillo de complicidad a Napoleón en carta al propio Enrique Umaña (1816) como al "infernal genio que presidía los destinos de la Francia", durante los años del criollo en Sorèze, y celebraba el actual "confinamiento" del corso en Santa Helena. León José permaneció interno en Sorèze durante toda su estadía en Francia. A pesar de que cultivó "con algún suceso el idioma francés, por supuesto, el inglés, la historia, la geografía, la aritmética, la física general, la equitación, la escrima, el clarinete, etc." ${ }^{34}$, no adelantó una carrera, como las que dispensaba entonces la École polythécnique (París), ni siguió el ejemplo de su hermano, nombrado corresponsal (1801) del Muséum National d'Histoire Naturelle, del Conseil de Mines y de la Sociedad de Ciencias, Bellas Letras y Artes de Burdeos, lo que confirma el apego de los Umaña a la tierra y su gusto moderado por la ciencia.

Umaña arribó a París en febrero de 1808. El 6 de mayo, Carlos IV abdicaba en favor de Napoleón, quien a su vez traspasó la corona de España a su hermano José. La opinión en

\footnotetext{
31 Real Cédula nombrando a Enrique Umaña Corregidor de Zipaquirá, Madrid, 29 de agosto 1807, en Alberto Corradine Angulo, "Noticia de algunos corregidores de Zipaquirá", Boletín de la Academia de Historia de Cundinamarca 3, no 5 (1977): 44-58.

32 García de la Guardia, Kalendario 1806, 96.

33 Oficio de José de Ezpeleta y Galdiano, Virrey del Nuevo Reino de Granada, a Antonio Porlier, marqués de Bajamar y ministro de Estado, Santafé, 19 de marzo, 1792. Archivo General de Indias (AGI). (Sevilla), Santafé, 557.

34 AFU, carta de L. J. Pontón a E. Umaña, La Habana, 30 de septiembre.
} 
España se vio sacudida por la violencia generalizada contra los hombres y las instituciones consideradas sagradas. El pueblo se indigno..$^{35}$ Justo en mayo, nuestro "corregidor provisto" por un gobierno derrocado regresó a Madrid para reunirse con su familia. Allí se enteró de la "pérdida de los intereses únicos con que contaba, por habérselos arrebatado los franceses". ${ }^{36}$ Este pasaje se refiere al despojo de las ganancias obtenidas por Umaña con las quinas y revela, sobre todo, que Umaña no tenía tratos con el invasor, ni pertenecía al partido de los afrancesados, y aún más, que le repugnaba la idea de ver extenderse en España las instituciones de la Revolución francesa (abolición de la Inquisición y de los bienes de manos muertas, entre otras). La antipatía de Umaña por Napoleón debió entonces convertirse en repulsión. En este sentido, la actitud de Umaña contrasta con la adhesión de Zea al reinado de José Bonaparte. En efecto, Zea representó al gobierno intruso como intendente de Málaga, y cuando las tropas napoleónicas abandonaron España, marchó a París con ellas y de París a las Antillas y de allí al Orinoco para unirse a Bolívar en 1816. ${ }^{37}$

Umaña se mantuvo leal a la monarquía. Ciertamente, los Borbones lo habían precipitado en las peores angustias en 1794. Luego habían venido los años interminables de prisión, en la flor de su juventud. Eran indescriptibles las aflicciones que le habían producido el dolor de sus padres, sentirse gravoso para ellos y tener que soportar las habladurías. A pesar de todo ello, el rey lo había cubierto de honores y de una experiencia de mundo impensable en la carrera de un patricio santafereño. Todo lo que era ahora se lo debía al rey. Esta vida de contrastes contribuyó sin duda a cautivar a María Vicenta Sánchez Manzaneque. Además, Umaña pensaba, y seguramente su familia política de arraigadas convicciones realistas, que España se encontraba ligada a un emperador y a un país cuya derrota era inevitable en un plazo más o menos corto. ${ }^{38}$ La ausencia del rey no podía ser sino temporal. España se recuperaría de la invasión francesa. La infidelidad de América era inimaginable.

Aduciendo el "atraso de su fortuna", Umaña solicitó y obtuvo, de orden y mandato de la Suprema Junta Central Gubernativa del Reino, ${ }^{39}$ el nombramiento de corregidor de Zipaquirá, el 30 de octubre de 1808. La Junta fue el órgano que acumuló los poderes ejecutivo y legislativo españoles durante la ocupación napoleónica. ${ }^{40}$ Se constituyó el 25 de septiembre de 1808, tras

35 Bottineau, Les Bourbons, 340.

36 Amaya y Varila, "Importancia del procesamiento".

37 Soto, Francisco Antonio Zea, 84. En El Mercurio, periódico estatal, Zea expresó su apoyo y admiración hacia el gobierno francés y sus científicos. Desde el primer número se declaró admirador de Napoleón y le llegó a considerar salvador de Francia. En cuanto a la política española, centra sus elogios en el Príncipe de la Paz. En Godoy reconoce al gran ministro que ha organizado el Ejército, los cosmógrafos de Estado, apoya los progresos de las ciencias y la instrucción pública. El ministro era, para el criollo, el que impulsaba las ideas liberales y a su alrededor se encontraba el grupo "avanzado" de los afrancesados. Era evidente que él se consideraba integrante de este grupo y, por tanto, no es de extrañar que tuviera la posición de defender los planes del gobierno de Godoy que en esta época se veían entorpecidos por el grupo tradicional. Véase Soto, Francisco Antonio Zea, 142.

38 Bottineau, Les Bourbons, 177.

39 Biblioteca Nacional de Colombia, Fondo Pineda, leg. 191, f. 604 recto y verso: "Licencia de embarco para don Enrique Umaña, su esposa y su hijo don Enrique Benito" (extendida por Rafael Orozco, Juez de Alzadas y Arribadas de Indias en Cádiz, 24 de enero, 1809)

40 El AFU conserva un interesante documento de EUB sobre la invasión napoleónica: "Por un barco que salió de Cádiz el 9 de marzo y llegó a la Guaira en 2 de abril de este año [de 1808], referente a carta que escribió a don Ignacio Umaña vecino de esta ciudad [de Santafé], su hijo [Enrique] se sabe: Que el 25 de enero se retiraron a Madrid los Reyes y Familia Real; que el 26 fue día terrible en Madrid, que en aquel día, de nueve de la mañana a dos de la tarde fueron ahorcados y arcabuceados setenta y tres personajes entre quienes se cuenta al Príncipe de la Paz (ahorcado) que había sido conducido desde la Alhambra de Granada el 9 de enero, doce guardias de corps, quince monteros, dos consejeros, dos ministros y otras varias personas de distinción; que se confiscaron sesenta millones a Godoy, que se embargaron cuatrocientos mil pesos más que Godoy había impuesto a favor de la Tudo; que la Plaza Mayor de Madrid fue ocupada por ciento veinte hombres de tropa a cuyo efecto había entrado el 17 de diciembre Tayllerand en Madrid y el 29, el Emperador de los franceses por Barcelona; que el 28 de enero se celebraron en Aranjuez los bodas de nuestro Príncipe de Asturias con la sobrina del Emperador. Santafé, 12 de mayo de 1808”. 
la victoria en la Batalla de Bailén y después de que el Consejo de Castilla declarase nulas las abdicaciones de Bayona y todas las actuaciones del gobierno francés, el 11 de agosto de 1808.

Umaña se posesionó el 30 de julio de 1809. ${ }^{41}$ Tenía 38 años. Era su primer empleo remunerado. $\mathrm{Su}$ perfil de burócrata era ciertamente singular, por su formación científica y voluntad reformista. Además, al no hallarse los intereses patrimoniales de su familia en las salinas y tampoco en la jurisdicción de Zipaquirá, el titular podía concentrarse al servicio del rey, como disponían las reformas borbónicas. Aunque de modesta remuneración, según se ha dicho, el cargo representaba un oportuno medio de lavar el apellido. Además, su ejercicio iba a permitirle al titular conocer la mecánica de la administración virreinal, antes de ocuparse de la administración de Tequendama. Al momento de su posesión, Umaña ya no tenía vínculo con la Expedición Botánica: su nombre no figura en el testamento de Mutis ni en la historia subsiguiente de aquella institución. ${ }^{42}$

Se ha demostrado que hasta los primeros meses de 1809 el primer instinto de los líderes criollos fue el de permanecer fieles a España, esperando una acomodación política con la metrópoli. ${ }^{43}$ Umaña, por su parte, era un hombre seguro para el Virrey Antonio Amar y Borbón (1742¿1826?). Cuando este último se sintió amenazado por los patriotas, procedió a una purga en el aparato burocrático del Estado que condujo a la destitución de varios corregidores (Socorro y Pamplona, entre otros) y Gobernadores (Cartagena y Chocó). Umaña permaneció en su puesto defendiendo la causa nacional contra el emperador de los franceses.

A estas alturas, dos perplejidades rondaban la felicidad del corregidor. Por una parte, representaba un gobierno de transición; de hecho, la Junta Central gobernará hasta el 30 de enero de 1811. Por otra, sus obligaciones con la administración de Tequendama se habían postergado, mientras durara su mandato.

41 Orjuela, Tributos de Zipaquirá, 198. Santiago Talero, modesto cronista de Zipaquirá, registra la llegada del corregidor y su familia a aquella parroquia en los siguientes términos: "El sábado 29 de julio de 1809 llegó a Zipaquirá el Dr. D. Enrique Umaña, y el día siguiente se recibió de Corregidor; y el miércoles 20 de octubre del mismo trajo su familia, y el jueves, viernes, sábado y domingo hubo toros". En "Cuaderno de sucesos acontecidos en ciertos días, meses y años", Zipaquirá, 1807-1819, 111 entradas, manuscrito citado por Orjuela, Minuta, 17-18). En la crónica de Talero se respira el aire cultural y político de Zipaquirá durante los años de la Primera República (1810-1815). Su labor escrituraria presenta aspectos sugestivos: ampliación de la función autor hacia sectores populares y emergencia de memorias locales diferentes a las tradicionales de curas, abogados y notables en general. Su texto es tanto más interesante cuanto que tiene continuidad y una cierta organización creciente en el tiempo. Talero no pertenecía a las redes de ilustrados criollos de la élite local, al punto de que el historiador Luis Orjuela (1850-1931), descendiente e incondicional de este último sector, lo califica de "crudo", aunque con "arraigadas creencias de cristiano viejo". La extrañeza de Orjuela es proporcional a la novedad de la figura de Talero en el espacio cultural de Zipaquirá. Talero debía simultanear su labor escrituraria con otra actividad u oficio artesanal. En la cita transcrita se percibe a un cronista que reorganiza sucesivamente sus notas. La descripción cronológica va ganando unidad temática en la fusión de dos momentos de la historia política y social del corregimiento: la llegada y posesión del corregidor y el arribo de su familia tres meses después. Talero abrigaba la convicción de que su terruño estaba viviendo sucesos nunca antes vistos que era indispensable consignar por escrito para la posteridad. La unidad de referencia es Zipaquirá y también, por cierto, la propia vida del cronista. En este sentido, llama la atención que la preocupación por los asuntos públicos corra parejas con un cierto interés por la propia individualidad. Por lo demás, Orjuela revela un irritante anacronismo cuando afirma: "Con júbilo se acogió en Zipaquirá el advenimiento del deseado funcionario ... Y el caso no era para menos. Era un criollo, y criollo de ideas de independencia probadas el que por buena suerte entraba a dirigir los negocios públicos en un corregimiento pocas veces confiado a la administración de manos no ligadas de nacimiento por la fidelidad para con España”. Orjuela, Tributos de Zipaquirá, 198.

42 Véase Guillermo Hernández de Alba, ed.,. Historia documental de la Real Expedición Botánica del Nuevo Reino de Granada después de la muerte de su director Don José Celestino Mutis 1808-1952 (Bogotá: Instituto Colombiano de Cultura Hispánica, 1986).

43 Víctor Manuel Uribe Urán, “ ¡Maten a todos los abogados!' Los abogados y el movimiento de Independencia en la Nueva Granada, 1809-1820", Historia y Sociedad, no 7 (2000): 32. 


\section{CONCLUSIÓN}

Tratando de huir de su destino, Umaña terminó acumulando en Europa una experiencia invaluable para el mantenimiento y ampliación del patrimonio de su familia. Forjó su carácter en la prisión; además, conoció personalmente en Francia el retorno y resarcimiento de aristócratas exilados en Londres y presenció la invasión de España y la abdicación de los Borbones. Con este trasfondo, la revolución de independencia será para él un juego de niños. En un sentido más general, con estas páginas se ha querido contribuir al estudio de la memoria de la Independencia, de la que no pueden marginarse quienes se mantuvieron fieles a la monarquía. El caso Umaña es sólo uno entre muchos otros que vale la pena conocer. El ciudadano colombiano tiene derecho a escuchar la voz de los vencidos. Umaña no fue un hombre de carrera ni como abogado, ni como mineralogista. A lo sumo podría decirse que su urgencia de ingresar a las filas de la burocracia estatal formaba parte de una estrategia familiar, destinada a liberarlo a él y a su familia de la fea mancha de 1794.

\section{FUENTES}

Archivo de la Familia Umaña (AFU), Bogotá-Colombia.

Archivo General de Indias (AGI), Sevilla-España. Santafé 557.

Archivo General de la Nación, Bogotá-Colombia. Notaría Segunda de Santafé, 1760-1808; República, Peticiones y Solicitudes.

Biblioteca Nacional de Colombia (BNC), Bogotá. Fondo Pineda, leg. 191.

\section{REFERENCIAS}

Amaya, José Antonio. "Cuestionamientos internos e impugnaciones desde el flanco militar a la Expedición Botánica". Anuario Colombiano de Historia Social y de la Cultura, no. 31 (2004): 75-118.

Amaya, José Antonio y Diego Armando Varila. "Importancia del procesamiento archivístico de los expedientes del Consejo de Guerra. Caso: Enrique Umaña (1771-1854) (Santafé, Nuevo Reino de Granada, 1816)". Ponencia leída en las XII Jornadas Archivísticas organizadas por la Universidad de Guanajuato y la Red Nacional de Archivos de Instituciones de Educación Superior (RENAIES). Guanajuato, 13-15 de junio, 2012.

Bottineau, Yves. Les Bourbons d'Espagne 1700-1808. París: Fayard, 1993.

Corradine Angulo, Alberto. "Noticia de algunos corregidores de Zipaquirá". Boletín de la Academia de Historia de Cundinamarca 3, no. 5 (1977): 44-58.

“De una carta de D. Camilo Torres a su padre D. Jerónimo Francisco de Torres, en la cual le informa de los sucesos políticos de Santa Fe en 1794". El Correo Nacional, 21 de julio, 1894.

Forero Benavides, Abelardo. "1794. La incubación de la Independencia". En Impresión y represión de los Derechos del Hombre, ed. Abelardo Forero Benavides. Bogotá: Ediciones Universidad de los Andes, 1967, 121-138.

García de la Guardia, Antonio. Kalendario manual y guía de forasteros en Santafé de Bogotá, capital del Nuevo Reyno de Granada, para el año de 1805. Santafé: Imprenta Real, 1804.

García de la Guardia, Antonio. Kalendario manual y guía de forasteros en Santafé de Bogotá año de 1806. Bogotá: Banco de la República, Edición facsimilar, 1988. 
González Bueno, Antonio. Antonio José Cavanilles (1745-1804). La pasión por la ciencia. Madrid: Fundación Jorge Juan, 2000.

Hernández de Alba, Guillermo. "Herencia y gloria acrecentadas: estudiantes y profesores de 1794", El Tiempo, 7 de julio, 1957.

Hernández de Alba, Guillermo. Historia documental de la Real Expedición Botánica del Nuevo Reino de Granada después de la muerte de su director Don José Celestino Mutis 1808-1952. Bogotá: Instituto Colombiano de Cultura Hispánica, 1986.

Ibáñez, Pedro María. “La conspiración de 1794”, El Tiempo, serie 3, no. 30, no. 984, 1914.

König, Hans-Joachim. En el camino hacia la nación. Nacionalismo en el proceso de formación del Estado y de la nación de la Nueva Granada, 1750 a 1856. Bogotá: Banco de la República, 1994.

López Piñero, José María. La obra botánica de Cavanilles. Valencia: Faximil Ediciones Digitales, 2004. http: / / www.faximil.com/descargas/ estudioicones.pdf

López Piñero, José María, y María Luz López Terrada. Bibliografía de Antonio José Cavanilles (1745-1804) y de los estudios de su vida y su obra, 3a. ed. Rev., s. l., s. e., s. f. http:/ / www.uv.es/ rseapv / Publicaciones/Cavanilles / RSEAP_Cavanilles_2A.pdf

Los Hechos. “1794-1894”. Los Hechos, serie 2, no. 156, 20 de julio, 1894.

Orjuela, Luis. Minuta histórica zipaquireña. Bogotá: Imprenta de La Luz, 1909.

Orjuela, Luis. Tributos de Zipaquirá para la Revolución de Independencia. Bogotá: Imprenta de La Luz, 1912.

Silva, Renán. Los ilustrados de Nueva Granada 1760-1808. Genealogía de una comunidad de interpretación. Medellín: Banco de la República y Fondo Editorial Universidad EAFIT, 2002.

Soto Arango, Diana. Francisco Antonio Zea. Un criollo ilustrado. Madrid: Ediciones Doce Calles, Colciencias, RUDECOLOMBIA, 2000.

Umaña Barragán, Enrique. Relación de los méritos y ejercicios literarios del doctor don Enrique Umaña Barragán, Madrid, 14 de mayo de 1807. Biblioteca Nacional de Colombia, Audiovisuales, VFDU1-401.

Uribe Urán, Víctor Manuel. “'¡Maten a todos los abogados!’. Los abogados y el movimiento de Independencia en la Nueva Granada, 1809-1820". Historia y Sociedad, no. 7 (2000): 7-48.

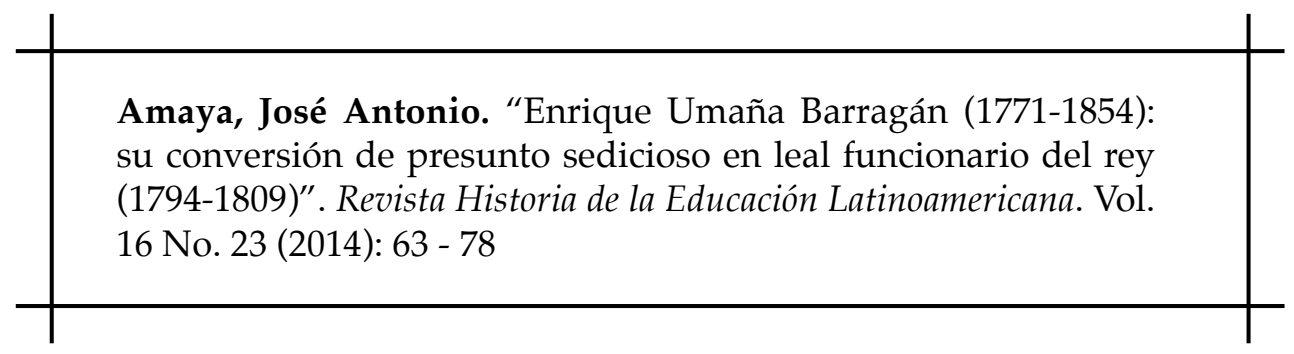




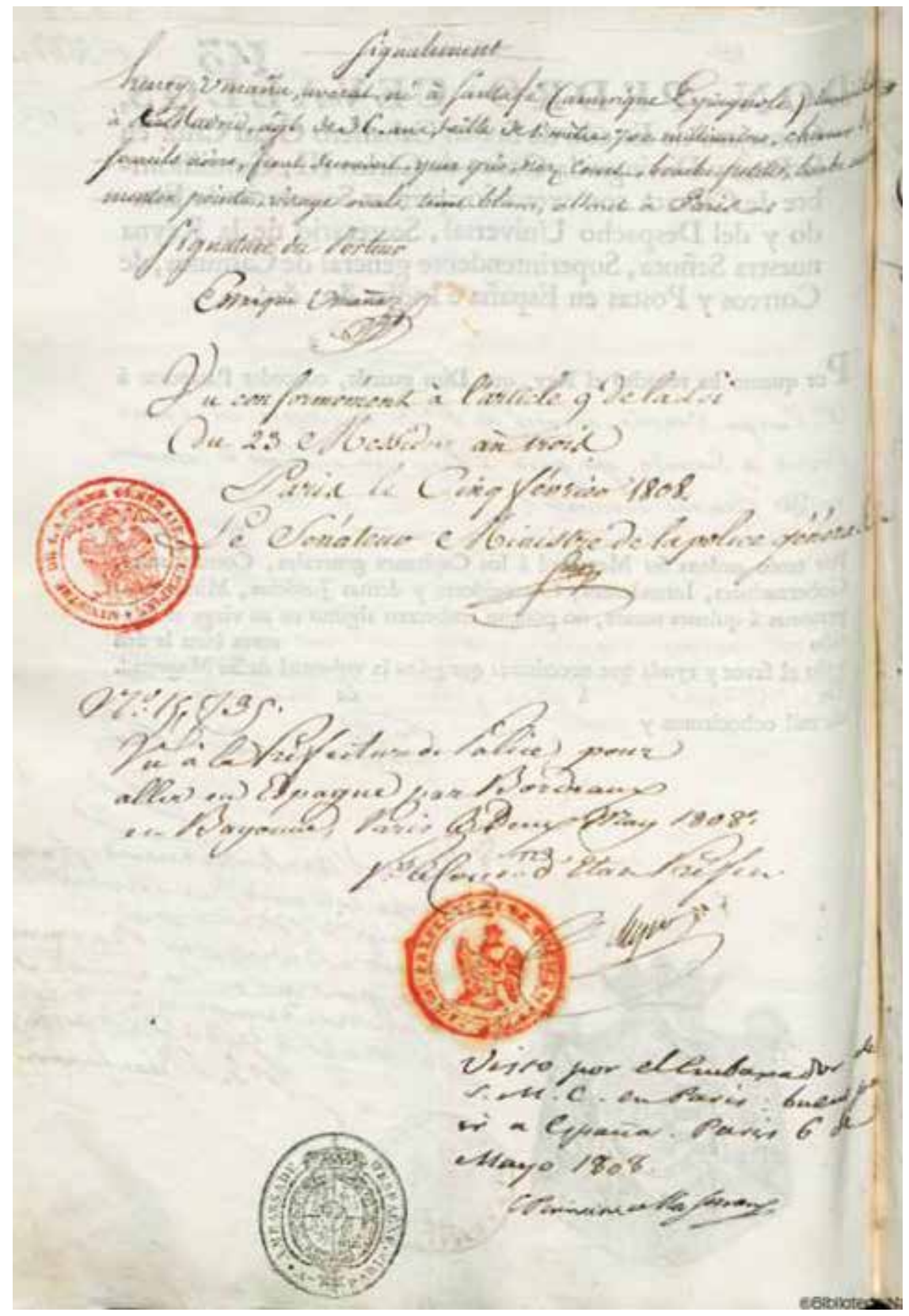

"Enrique Umaña, abogado, natural de Santafé (américa Española), domiciliado en Madrid, de 36 años de edad, estatura 1 metro, 700, milímetros, cabello y cejas negras, frente amplia, ojos grises, nariz pequeña, boca pequeña, barba espera, mentón prominente, rostro ovalado, piel blanca..." . "Henry Umaña, avocat, né à Santafé (amériqueEspagnole), domicilié à Madrid, âgé de 36 ans, taille 1 mètre 700, millimetres, cheveux et sourcilsnoirs, frontdécouvert, yeux gris, nezcourt, bouchepetite, barbe nourrie, mentón pointu, visage ovale, teintblanc...). Filiación de Enrique Umaña Barragán, estampada en su pasaporte y levantada en París el 5 de febrero de 1808. Original en Biblioteca Nacional de Colombia. Fondo Anselmo Pineda. "Documentos relativos a la vida y hechos públicos del Doctor Don Enrique Umaña". R. M. 00191, Documentos Históricos, t. 2, , f. 603v. Traducción de José Antonio Amaya. 